\title{
Academic fraud: perspectives from a lifelong anesthesia researcher
}

\author{
W. Alan C. Mutch, MD
}

Published online: 4 June 2011

(C) Canadian Anesthesiologists' Society 2011

Science and medicine have been beset by an increasing incidence of fraud detected in academic research. ${ }^{1-3}$ Historically, academic fraud involving either ethical and/or scientific misconduct has often been associated with high profile journals. ${ }^{4-8}$ A number of these high profile cases have involved anesthesiologists. ${ }^{9,10}$ One well-publicized case of academic fraud resulted in the imprisonment of anesthesiologist, Dr. Scott Reuben, who was at the centre of more than 20 falsified studies in multimodal pain therapy. ${ }^{11}$ More recently, a joint statement from the editors-inchief of 16 anesthesia, perioperative, and critical care journals has outlined the group retraction of 88 articles authored or co-authored by another anesthesiologist, Dr. Joachim Boldt. ${ }^{12}$ The large number of article retractions over the past several years brings into question whether there is a specialty-related propensity for anesthesiologists to be tempted to commit academic fraud, or are the recent exposures merely a statistical blip? Steen ${ }^{13}$ argues convincingly that medical fraud is increasing in general. However, he also notes that Dr. Reuben was responsible for $25 \%$ of the fraudulent papers retracted in 2009 (a total of 13 of his papers that year) as listed on PubMed. ${ }^{13}$ Unfortunately, given the number of retractions

Dr. Mutch is the 2011 recipient of the Canadian Anesthesiologists' Society Research Recognition Award.

W. A. C. Mutch, MD

Department of Anesthesia and Perioperative Medicine,

University of Manitoba, Winnipeg, MB, Canada

W. A. C. Mutch, MD ( $\square)$

Anesthesia Research Laboratory, A504 Chown Building,

753 McDermot Avenue, Winnipeg, MB R3E 0W3, Canada

e-mail: amutch@cc.umanitoba.ca attributable to Dr. Boldt (a number likely to rise), our specialty will remain in the limelight.

There are many different forms of academic fraud, ranging from text or data plagiarism to ghostwriting, to data and image manipulation, to data and image fabrication. The first problem, text plagiarism, is now being addressed very actively as new and more powerful software programs compare submitted manuscript text to existing published works both in journals and theses. ${ }^{14}$ In the case of Dr. Reuben's articles, the retractions exemplify the more egregious examples relating to data manipulation and fabrication. In the case of Dr. Boldt, the 88 articles identified on the editors' joint statement were retracted due to lack of evidence of Institutional Research Ethics Board approval, which constitutes ethical misconduct in research. There are ongoing investigations to assess the scientific integrity of these articles, since the first article retraction was on the basis of data falsification. ${ }^{10}$ In view of the above, it is important to consider the risk that academic fraud may impose on anesthesia, to contemplate why our specialty may be at greater risk in some circumstances, and to suggest potential solutions.

\section{The perils of academic fraud}

Academic fraud imperils medicine in general, and the consequences can be substantial. As trust in medicine is seriously harmed, the integrity of the research enterprise is damaged, and in certain circumstances, fraudulent research can directly or indirectly injure patients. ${ }^{1,15,16}$ Grievous loss of personal stature can occur, often to previously wellregarded colleagues. A department can be seriously compromised, a university tainted, and a specialty wounded. Until discovery, fraudulent research can move the research 
enterprise of a specialty in the wrong direction and can squander large sums of scarce research dollars. One of our sacred trusts is based on the Hippocratic Oath - primum non nocere - "First, do no harm". With academic fraud, multiple patients can be injured. Any anesthesia-related fraud is compounded in this context. It is well known that anesthesia funding from the major granting agencies is scant at the national level. ${ }^{17,18}$ Thus, a disproportionate incidence of fraud may have a significant negative impact on our specialty's credibility.

\section{The researcher type}

Almost without exception, academic fraud is committed by ambitious, highly driven, motivated individuals, and by and large, those interested in pursuing a research career possess these characteristics. So what distinguishes the conscientious, honest researcher from the fraudulent researcher? For each occurrence of fraud, it is likely that there were individual circumstances that led the researcher astray. From his study of research fraud, Steen ${ }^{19}$ has identified the following characteristics of the deliberately fraudulent scientist: a) a first author or a senior author who is a repeat offender; b) more frequently publishes with at least one co-author who also has fraudulent publications; c) has a greater number of co-authors-suggested as a means to diffuse responsibility; d) targets journals with a high impact factor; e) has delayed retraction compared with papers rejected solely on account of errors, and f) perhaps surprisingly, is associated with papers originating from the United States. It seems that fraud is most likely to occur early in a researcher's career when the individual is "establishing her/his presence". It is less likely to occur in mid-career (unless under considerable stress for research grants), but potentially reoccurs later in a career when the researcher's reputation is already established - an established reputation can act as a leverage to increase publication output. Such use of leverage appears evident in the increasing ratio of retractions: in the latter years of Dr. Reuben's publication history as recorded in PubMed, at least $40 \%$ of his papers published yearly from 2004 to 2008 were retracted publications, a much higher percentage than in his earlier years with publications documented back to 1991.

\section{Problems real or potential relating to anesthesia and perioperative medicine}

Research in anesthesia and perioperative medicine clearly covers a full spectrum from benchtop studies to examination of administrative databases. Anesthesia is not unique in this regard. As with other specialties, investigators must be vigilant with regard to the relationships and real or perceived conflicts of interest with pharmaceutical companies and manufacturers of medical devices. However, one of the most important research avenues in anesthesia involves randomized controlled trials and observational studies of patients undergoing operative procedures. We routinely engage in a collaborative enterprise with surgical and perioperative physician colleagues. Co-authorship is shared routinely in this regard. In all circumstances, it is important that co-authorship is earned and not awarded simply as a means to facilitate academic studies. Major journals have provided an important monitoring service here by insisting that each author attest to the nature of her/ his contribution to a given study. In this context, falsification of contribution by means of a signature could be viewed as an error of commission rather than an error of omission. The multiple retractions of work co-authored by Dr. Boldt are instructive here as well. It requires suspension of disbelief that errors of commission did not occur, at least for some repeat co-authors. A comprehensive investigation of Dr. Reuben's co-authors has been undertaken ${ }^{20}$; such remains to be undertaken for the body of work associated with Dr. Boldt.

Many of our perioperative studies now involve information retrieval from increasingly sophisticated data acquisition systems that are able to procure vast data streams, often at an unprecedented level of fidelity. I have used such systems for over 25 years, and from my perspective these may represent a double-edged sword for the anesthesia researcher-as an underappreciated risk to data integrity. Unless a dedicated technician or research trainee is recording events associated with the perioperative course in real time, it can be very difficult to correlate changes in hemodynamic variables or depth of anesthesia at a later date. At best, this can make data interpretation problematic, or at worst, it can make data interpretation subject to manipulation and thus falsification.

\section{Problems of opportunity}

For the researcher or research administrator, opportunities often arise to expand one's curriculum vitae easily. While working as a Medical Research Fellow in Copenhagen Denmark in the early 1980s, I was afforded the opportunity for multiple co-authorships based on editing manuscripts for scientists who were writing in English as their second language. After careful consideration, I chose not to co-author any article I edited while there. In my view, if I were examined in the future, I would have difficulty defending co-authorship on a paper examining "the nature of movement of pinocytotic vesicles to the abluminal 
vascular endothelial surface in the hagfish". While an individual may be afforded an opportunity for an easy increase in co-authorships, and the individual's national and international colleagues may be impressed with such measured productivity, one's local colleagues are perhaps best positioned to appreciate a researcher's skill set. In light of this "problem of opportunity", researchers should be guided by the International Committee of Medical Journal Editors (http://www.icmje.org) criteria for authorship and the related guidelines provided in the Instructions for Authors in the respective journals.

\section{Solutions to the problem of academic fraud}

How can we prevent academic fraud in the specialty of anesthesia or, in a larger context, research fraud in medicine in general? While this problem may never be eradicated completely, the problem can be limited by good mentoring, appropriately trained and diligent support staff, blinded assessment of data, data review by all investigators, a consensus agreement on data interpretation, a vigorous and independent Research Office, effective internal and external committees to assess adherence to protocols, and strong departmental leadership. Further, a call has been made in the recent past for a National Research Integrity Agency. ${ }^{21}$ We must encourage renewed respect for rigorous conduct of research and accuracy and transparency of scientific reporting, and we must provide bona fide review authority to our Research Offices over research protocols and grant applications. We should also encourage robust statistical consultations. Miller has highlighted the requirement for researchers to be conversant with Good Clinical Practice (GCP) Guidelines and to follow Tripartite Council Policy. ${ }^{22}$ At the level of a journal's editorial office, if an editor or reviewer is suspicious about a manuscript's veracity, guidance is offered on the appropriate course of action by the Committee on Publication Ethics (COPE) ${ }^{23}$ When research fraud is suspected, the problem must be dealt with head on, ideally through an established standing committee. Editorial offices are not investigative bodies, nor should they be. The responsibility to ensure research integrity lies at the level of the institutions where the research was conducted. Internal review is fraught with inadequate assessment and the failures are well documented. ${ }^{6,15}$ Established procedures are especially important for the senior researcher with an extensive reputation. Being the whistleblower is not pleasant, but the consequences to the research enterprise, the reputation of the department and the university, and the risk of patient injury are too great to ignore. ${ }^{24}$ However, as highlighted by the Olivieri/Apotex case, it is sobering to note that problems persist in being a whistleblower. ${ }^{25}$
In Canada, at a national level, an important monitoring group has been struck for anesthesia research. The Perioperative Anesthesia Clinical Trials (PACT) group is now in place to aid anesthesia research in Canada. ${ }^{26}$ This group, comprising nationally prominent anesthesia researchers, will provide important mentoring and supervision of anesthesia clinical trials originating from our academic departments. Meritorious projects will be reviewed by this committee with active involvement and supervision of the selected projects. Such a body can potentially make an important contribution towards maintaining the integrity of the anesthesia research enterprise.

Many in the field of anesthesia worry about the "deprofessionalization" of our specialty. ${ }^{27}$ One of the most important ways to maintain a high level of professionalism is to embrace an active research enterprise in our chosen field. Recent cases of academic fraud have compromised part of the body of scientific knowledge in our specialty, trust relationships at a number of levels, and the reputation of several institutions. We must be engaged in ensuring that our research is novel, scientifically valid and important, and undertaken with honesty and accuracy. Reasoned interpretation without reporting bias, absence of suppression of safety data, and accurate reporting of adverse events are critical to sound scientific conduct. Our patients, whose clinical care depends on a body of knowledge derived from an accurate scientific record, deserve no less.

\section{La fraude scientifique: quelques considérations d'un chercheur en anesthésie aguerri}

La science et la médecine sont en proie à une augmentation des cas de fraude découverts dans la recherche universitaire. ${ }^{1-3}$ Historiquement parlant, la fraude scientifique par malhonnêteté éthique et/ou scientifique a souvent été associée aux revues réputées. ${ }^{4-8}$ Des anesthésiologistes ont été mêlés à plusieurs de ces fameux cas de fraude. ${ }^{9,10} \mathrm{Un}$ cas dont on a beaucoup parlé a entraîné l'incarcération d'un anesthésiologiste, le Dr Scott Reuben, qui était l'un des principaux auteurs de plus de 20 études falsifiées dans le traitement multimodal de la douleur. ${ }^{11}$ Plus récemment, une déclaration conjointe des rédacteurs en chef de 16 revues d'anesthésie, de soins périopératoires et de soins intensifs a mis en exergue la rétraction groupée de 88 articles rédigés ou co-rédigés par un autre anesthésiologiste, le Dr Joachim Boldt. ${ }^{12}$ Le nombre important de rétractions d'articles au cours des dernières années soulève une question qui mérite notre attention: existe-t-il une propension liée à notre spécialité qui incite les 
anesthésiologistes à céder à la tentation de commettre une fraude scientifique, ou les récents cas rendus publics ne sont-ils qu'un simple accident statistique? Steen ${ }^{13}$ soutient, de façon fort convaincante, que les cas de fraude médicale sont en augmentation en général. Toutefois, il note également que le Dr Reuben était responsable de $25 \%$ des articles frauduleux rétractés en 2009 (un total de 13 de ses articles cette année-là) tels qu'ils sont énumérés sur PubMed. ${ }^{13}$ Malheureusement, en raison du nombre de rétractions attribuables au Dr Boldt (un nombre qui ira probablement croissant), notre spécialité demeurera sous les feux de la rampe.

Il existe de nombreuses formes de fraude scientifique, allant du plagiat textuel ou de données au recours à un auteur anonyme, en passant par la manipulation des données et des images, voire à la fabrication de données ou d'images. Le premier problème, le plagiat textuel, fait désormais l'objet d'examens minutieux grâce à l'apparition de nouveaux logiciels de plus en plus puissants qui permettent de comparer les manuscrits soumis aux travaux existants publiés dans des revues ou des thèses. ${ }^{14}$ Dans le cas des articles du Dr Reuben, les rétractions illustrent certains des exemples les plus flagrants liés à la manipulation et à la fabrication de données. Dans le cas de Dr Boldt, les 88 articles cités dans la déclaration conjointe des rédacteurs en chef ont été rétractés parce qu'il était impossible de prouver l'obtention de l'approbation du Comité d'éthique de la recherche de l'institution où il pratiquait, ce qui, en recherche, est considéré comme éthiquement malhonnête. Des enquêtes sont en cours pour évaluer l'intégrité scientifique de ces articles, étant donné que la première rétraction se fondait sur un cas de falsification de données. ${ }^{10}$ Pour toutes ces raisons, il est important de tenir compte du risque que la fraude scientifique pourrait faire courir à l'anesthésie, d'essayer de comprendre pourquoi notre spécialité pourrait être particulièrement à risque dans certains cas, et de proposer des solutions possibles à ce problème.

\section{Les périls de la fraude scientifique}

La fraude scientifique met en péril la médecine en général, et ses conséquences peuvent être dramatiques. En effet, la fraude scientifique entraîne une érosion de la confiance en la médecine, une dégradation de l'intégrité liée à la réalisation de recherches et, dans certains cas, elle pourrait même nuire, directement ou indirectement, aux patients. ${ }^{1,15,16}$ Une importante chute de statut personnel peut survenir, souvent à l'encontre de collègues auparavant tenus en haute estime. Un département peut être gravement compromis, la réputation d'une université entachée, et une spécialité blessée. Avant qu'elles ne soient révélées comme telles, les recherches frauduleuses peuvent faire avancer les recherches d'une spécialité dans la mauvaise direction et peuvent dilapider des sommes importantes de l'argent - déjà rare - destiné à la recherche. L'une de nos responsabilités sacrées se fonde sur le serment d'Hippocrate - primum non nocere - « En premier lieu, ne pas nuire ». En cas de fraude scientifique, de nombreux patients peuvent être lésés. Dans un tel contexte, toute fraude liée à l'anesthésie a un effet multiplicateur. Il est bien connu que le financement de l'anesthésie provenant des principales agences de financement est maigre au niveau national. ${ }^{17,18}$ Une incidence disproportionnée de fraude pourrait par conséquent avoir un impact négatif majeur sur la crédibilité de notre spécialité.

\section{Le type de chercheurs}

À quelques rares exceptions près, la fraude scientifique est commise par des personnes ambitieuses, très déterminées et motivées et, dans l'ensemble, les personnes intéressées par une carrière en recherche possèdent toutes ces caractéristiques. Dès lors, qu'est-ce qui distingue le chercheur consciencieux et honnête du chercheur frauduleux? Pour chaque cas où une fraude est commise, il est probable que des circonstances particulières ont détourné le chercheur du droit chemin. En étudiant le phénomène de la fraude en recherche, $\operatorname{Steen}^{19}$ a pu identifier les caractéristiques suivantes chez le scientifique sciemment frauduleux : a) un premier auteur ou un auteur senior qui est un récidiviste; b) un auteur qui publie fréquemment avec au moins un co-auteur qui possède également des publications frauduleuses; c) une personne qui utilise un nombre très élevé de co-auteurs - ceci indique une façon potentielle de diffuser la responsabilité; d) un auteur qui cible les revues avec un facteur d'impact élevé; e) la rétraction de ses articles est retardée par rapport aux articles rejetés seulement en raison d'erreurs; et f) de façon surprenante peut-être, l'auteur est associé à des articles provenant des États-Unis. Il semble que la fraude soit plus fréquente au début de la carrière d'un chercheur, lorsque la personne «établit sa présence ». La fraude est moins probable en milieu de carrière (sauf en cas de stress majeur pour l'obtention de bourses de recherche), mais elle peut potentiellement survenir à nouveau plus tard dans la carrière d'un individu, lorsque sa réputation de chercheur est déjà établie; en effet, une réputation établie peut constituer un levier pour augmenter le nombre de publications. Ce type de levier est évident dans le ratio croissant de rétractions : dans les années plus récentes de l'historique de publication de Dr Reuben tel qu'établi par PubMed, au moins $40 \%$ des articles publiés entre 2004 et 2008 étaient des publications non rétractées, un 
pourcentage bien plus élevé que dans ses premières années de carrière, si l'on considère ses publications documentées en remontant jusqu'en 1991.

\section{Les problèmes réels et potentiels liés à l'anesthésie et à la médecine périopératoire}

Il existe clairement toute une gamme de recherches réalisées dans les domaines de l'anesthésie et de la médecine périopératoire, qui vont des études réalisées en laboratoire à l'analyse des bases de données administratives. L'anesthésie n'est pas la seule spécialité dans une telle situation. Tout comme dans le cas d'autres spécialités, les chercheurs doivent faire preuve de vigilance quant aux relations et aux conflits d'intérêt, qu'ils soient réels ou perçus, avec les compagnies pharmaceutiques et les fabricants d'appareils médicaux. Cependant, l'une des voies de recherche les plus importantes en anesthésie est celle des études randomisées contrôlées et des études observationnelles portant sur des patients subissant des interventions en salle d'opération. Nous collaborons fréquemment avec nos collègues de chirurgie et de médecine périopératoire. La co-paternité est, par conséquent, fréquemment partagée. Indépendamment des circonstances, il est important que la co-paternité soit méritée et non pas simplement offerte comme un moyen de faciliter les études universitaires. Les revues les plus importantes ont mis en place un outil de surveillance important dans ce domaine en exigeant que chaque auteur fasse état de la nature de sa contribution à une étude donnée. Dans un tel contexte, la falsification de la contribution par une signature pourrait être interprétée comme une erreur de commission plutôt qu'une erreur d'omission. Les rétractions multiples de travaux co-rédigés par le Dr Boldt sont également un bon exemple dans le cas présent. Ces rétractions impliquent que l'incrédulité soit suspendue face au fait que des erreurs de commission ne sont pas survenues - pour le moins pour certains co-auteurs dont le nom apparaît dans plusieurs études. Une enquête complète portant sur les co-auteurs de Dr Reuben a été entreprise ${ }^{20}$; une telle enquête doit encore être entreprise pour les travaux associés au Dr Boldt.

Nombre de nos études périopératoires se fondent désormais sur des informations récupérées dans des systèmes d'acquisition de données de plus en plus perfectionnés, lesquels sont capables de fournir de vastes flux de données et ce, à un niveau de fidélité souvent inégalé. Selon moi, l'utilisation de tels systèmes pour plus d'un quart de siècle pourrait avoir favorisé l'apparition d'une épée à double tranchant pour le chercheur en anesthésie, et ces systèmes pourraient représenter un risque sous-estimé au maintien de l'intégrité des données. À moins qu'un technicien dédié ou qu'un stagiaire de recherche ne soit présent pour prendre note des événements associés au déroulement périopératoire en temps réel, il peut être très difficile de corréler des changements au niveau des variables hémodynamiques ou de la profondeur de l'anesthésie à une date ultérieure. Dans le meilleur des cas, cela peut rendre difficile l'interprétation des données complexe; dans le pire, l'interprétation des données peut alors être victime de manipulation et, par conséquent, de falsification.

\section{Les problèmes d'occasion}

Pour le chercheur ou l'administrateur de recherche, les occasions d'allonger facilement son curriculum vitae sont nombreuses. Alors que je travaillais comme stagiaire en recherche médicale à Copenhague, au Danemark, au début des années 1980, on m'a proposé de participer en tant que co-auteur à plusieurs articles si je consentais à éditer les manuscrits de scientifiques écrivant en anglais, leur deuxième langue. Après mûre réflexion, $\mathrm{j}$ 'ai préféré ne pas être un co-auteur des articles que j'ai corrigés au cours de mon séjour. Selon mon raisonnement, si mes recherches devaient être examinées à l'avenir, j'aurais des difficultés à justifier la co-paternité d'un article examinant « la nature du mouvement des vésicules pinocytotiques vers la surface endothéliale vasculaire abluminale chez la myxine ». Bien qu'une personne puisse avoir des occasions d'augmenter avec peu d'effort ses publications par le biais d'articles co-rédigés, et que les collègues de cette personne, aux niveaux national et international, puissent être impressionnés par une telle productivité, les collègues locaux de cette personne sont peut-être les mieux placés pour évaluer l'ensemble des compétences d'un chercheur. À la lumière de ce «problème de l'opportunité », les chercheurs devraient fonder leur jugement sur les critères du Comité international des rédacteurs de revues médicales (ICMJE - http://www.icmje.org) définissant un auteur et sur les directives associées disponibles dans les Directives aux auteurs des revues respectives.

\section{Quelques solutions au problème de fraude scientifique}

Comment peut-on prévenir la fraude scientifique en anesthésie, ou, si l'on voit plus grand, la fraude dans les recherches médicales en général? Bien qu'il soit possible que le problème ne soit jamais complètement éradiqué, il peut toutefois être limité grâce aux éléments suivants : un bon mentorat, un personnel de soutien bien formé et appliqué, l'évaluation en aveugle des données, la révision des données par tous les chercheurs, un accord consensuel sur l'interprétation des données, un Bureau de la recherche dynamique et indépendant, des comités internes et externes 
efficaces pour évaluer le respect des protocoles, ainsi qu'un leadership départemental fort. En outre, un appel à la création d'une Agence nationale pour l'intégrité de la recherche a récemment été lancé. ${ }^{21}$ Nous devons favoriser un respect renouvelé envers la réalisation rigoureuse de recherches ainsi qu'envers la précision et la transparence de la rédaction scientifique; en outre, nous devons proposer un organisme sérieux de supervision à nos Bureaux de recherche pour les protocoles de recherche et les candidatures aux subventions. Nous devrions également encourager les consultations statistiques rigoureuses. Le Dr Miller a souligné la nécessité, pour les chercheurs, de bien connaître les Directives des Bonnes pratiques cliniques (BPC) et de suivre la Politique tripartite des Conseils. ${ }^{22} \mathrm{Au}$ niveau du bureau de rédaction du Journal, si un rédacteur ou un réviseur a des soupçons quant à la véracité d'un manuscrit, le Comité sur l'éthique de publication (COPE) offre des recommandations sur la marche à suivre. ${ }^{23}$ Malheureusement, lorsqu'on soupçonne qu'il y a fraude scientifique, le problème doit être abordé de front, dans l'idéal via un comité permanent établi. Les bureaux de rédaction ne sont pas des organismes d'enquête, et ils ne devraient pas l'être. La responsabilité d'assurer l'intégrité de la recherche incombe aux institutions dans lesquelles les recherches sont menées. La surveillance à l'interne est pleine de cas d'évaluations inadaptées et les échecs d'un tel système sont bien documentés. ${ }^{6,15}$ Des processus bien établis sont tout particulièrement importants pour le chercheur senior qui jouit d'une plus grande réputation. Il n'est pas plaisant d'être le délateur, mais les conséquences pour la recherche, la réputation du département et de l'université, et le risque de nuire aux patients sont trop importants pour être ignorés. ${ }^{24}$ Cependant, comme le démontre le cas Olivieri/Apotex, il convient de garder à l'esprit que des problèmes persistent lorsqu' on veut tirer la sonnette d'alarme. ${ }^{25}$

$\mathrm{Au}$ Canada, au niveau national, un important groupe de surveillance a été établi pour la recherche en anesthésie. Le groupe PACT (Perioperative Anesthesia Clinical Trials) est désormais en place pour aider la recherche en anesthésie au Canada. ${ }^{26}$ Ce groupe est composé d'éminents chercheurs en anesthésie au pays; son rôle sera de fournir un important mentorat ainsi que de superviser les études cliniques en anesthésie provenant de nos départements académiques. Les projets dignes de mérite seront passés en revue par ce comité, qui s'impliquera activement dans la supervision des projets sélectionnés. Un tel organisme pourrait potentiellement contribuer de façon significative au maintien de l'intégrité de la recherche en anesthésie.

Nombreux sont ceux qui, dans le domaine de l'anesthésie, s'inquiètent de la «déprofessionnalisation » de notre spécialité. ${ }^{27}$ L'une des façons les plus importantes de maintenir un niveau élevé de professionnalisme est de s'engager activement dans la recherche dans notre domaine de prédilection. Les récents cas de fraude scientifique ont ébranlé une partie du corpus de connaissances scientifiques de notre spécialité, les relations de confiance à plusieurs niveaux, et la réputation de plusieurs institutions. Nous devons adopter une attitude proactive afin de garantir que nos recherches sont innovantes, scientifiquement valides et importantes, et qu'elles sont entreprises avec honnêteté et précision. L'interprétation raisonnée, sans biais dans la présentation des résultats, en l'absence de suppression des données de sécurité, et la présentation exacte des événements défavorables sont des éléments essentiels à une conduite scientifique saine. Nos patients, dont les soins cliniques dépendent d'un corpus de connaissances dérivées d'une présentation précise des résultats scientifiques, le méritent.

Competing interests None declared.

\section{References}

1. Baerlocher MO, O'Brien J, Newton M, Gautam T, Noble J. Data integrity, reliability and fraud in medical research. Eur $J$ Intern Med 2010; 21: 40-5.

2. Marshall E. Scientific misconduct. How prevalent is fraud? That's a million-dollar question. Science 2000; 290: 1662-3.

3. Catano VM, Turk J. Fraud and misconduct in scientific research: a definition and procedures for investigation. Med Law 2007; 26: 465-76.

4. Anonymous. Retraction-Ileal-lymphoid-nodular hyperplasia, nonspecific colitis, and pervasive developmental disorder in children. Lancet 2010; 375: 445.

5. Braunwald E. On analyzing scientific fraud. Nature 1987; 325: 215-6.

6. Culliton BJ. Coping with fraud: the Darsee case. Science 1983; 220: 31-5.

7. Relman AS. Lessons from the Darsee affair. N Engl J Med 1983; 308: 1415-7.

8. Felig $P$. Data on insulin binding in growth hormone deficiency are questionable. N Engl J Med 1980; 303: 1120.

9. Shafer SL. Notice of retraction. Anesth Analg 2009; 108: 1350.

10. Shafer SL. Notice of retraction. Anesth Analg 2010; 111: 1567.

11. Tanne $J H$. US pain expert faces prison after pleading guilty to research fraud. BMJ 2010; 340: c1207.

12. Editors-in-Chief. Editors-in-Chief Statement Regarding Published Clinical Trials Conducted Without IRB Approval by Joachim Boldt. Canadian Journal of Anesthesia, 2011. Available from URL: http://www.springer.com/medicine/anesthesiology/ journal/12630 (accessed April 2011).

13. Steen $R G$. Retractions in the scientific literature: is the incidence of research fraud increasing? J Med Ethics 2011; 37: 249-53.

14. Shafer SL. You will be caught. Anesth Analg 2011; 112: 491-3.

15. Sheehan JG. Fraud, conflict of interest, and other enforcement issues in clinical research. Cleve Clin J Med 2007; 74(Suppl 2): S63-7. discussion S68-9.

16. Jureidini JN, McHenry LB. Conflicted medical journals and the failure of trust. Account Res 2011; 18: 45-54.

17. Schwinn DA, Balser JR. Anesthesiology physician scientists in academic medicine: a wake-up call. Anesthesiology 2006; 104: $170-8$. 
18. Kuczkowski KM. "Code blue": monitoring the vital signs of academic anesthesia. Ann Fr Anesth Reanim 2004; 23: 838-9.

19. Steen $R G$. Retractions in the scientific literature: do authors deliberately commit research fraud? J Med Ethics 2011; 37: 113-7.

20. White PF, Rosow CE. Shafer SL; Editorial Board of Anesthesia and Analgesia. The Scott Reuben saga: one last retraction. Anesth Analg 2011; 112: 512-5.

21. Kondro W, Hebert PC. Research misconduct? What misconduct? CMAJ 2007; 176: 905, 907.

22. Miller DR. Publication fraud: implications to the individual and to the specialty. Curr Opin Anaesthesiol 2011; 24: 154-9.

23. Committee on Publication Ethics (COPE). Promoting Integrity in Research Publication. Available from URL: http://publication ethics.org 2011 (accessed April 2011).
24. Kesselheim AS, Studdert DM, Mello MM. Whistle-blowers' experiences in fraud litigation against pharmaceutical companies. N Engl J Med 2010; 362: 1832-9.

25. Rhodes $R$, Strain JJ. Whistleblowing in academic medicine. J Med Ethics 2004; 30: 35-9.

26. Hall R, Beattie $S$, Cheng D, et al. Can we develop a Canadian Perioperative Anesthesiology Clinical Trials group? Can J Anesth 2010; 57: 1051-7.

27. Wong A. From the front lines: a qualitative study of anesthesiologists' work and professional values. Can J Anesth 2011; 58: 108-17. 